\title{
Structure and Dynamics of Photosystem II Light-Harvesting Complex Revealed by High-Resolution FTICR Mass Spectrometric Proteome Analysis
}

\author{
Dmitry Galetskiy, ${ }^{a}$ Iuliana Susnea, ${ }^{a}$ Verena Reiser, ${ }^{\text {b,* }}$ Iwona Adamska, ${ }^{\text {b }}$ \\ and Michael Przybylski ${ }^{a}$ \\ a Laboratory of Analytical Chemistry and Biopolymer Structure Analysis, Department of Chemistry, \\ University of Konstanz, Konstanz, Germany \\ ${ }^{b}$ Department of Physiology and Plant Biochemistry, Department of Biology, University of Konstanz, \\ Konstanz, Germany
}

Structure and dynamics of membrane-bound light-harvesting pigment-protein complexes (LHCs), which collect and transmit light energy for photosynthesis and thereby play an essential role in the regulation of photosynthesis and photoprotection, were identified and characterized using high-resolution Fourier transform ion cyclotron resonance mass spectrometry (FTICR-MS). LHCs from photosystem II (LHCII) were isolated from the thylakoid membrane of Arabidopsis thaliana leaves after light stress treatment using sucrose density gradient centrifugation, and separated by gel-filtration into L.HCII subcomplexes. Using reversed-phase high-performance liquid chromatography and two-dimensional (2D) gel electrophoresis, the LHCII proteins, Lhcb1-6 and fibrillins, were efficiently separated and identified by FTICR-MS. Some of the LHCII subcomplexes were shown to migrate from photosystem II to photosystem I as a result of short-term adaptation to changes in light intensity. In the mobile LHCII subcomplexes, decreased levels of fibrillins and a modified composition of LHCII protein isoforms were identified compared to the tightly bound LHCII subcomplexes. In addition, FTICR-MS analysis revealed several oxidative modifications of LHCII proteins. A number of protein spots in 2D gels were found to contain a mixture of proteins, illustrating the feasibility of high-resolution mass spectrometry to identify proteins that remain unseparated in 2D gels even upon extended $\mathrm{pH}$ gradients. (J Am Soc Mass Spectrom 2008, 19, 1004-1013) (C 2008 American Society for Mass Spectrometry

$\mathrm{P}$ hotosystems I and II (PSI and PSII) represent the two basic types of photosynthetic reaction centers in cyanobacteria, green algae, and higher plants: type 1 using FeS clusters as electron acceptors and type 2 using pheophytins and quinones, respectively. Photosynthetic processes are initiated by light absorption through chlorophyll excitation in light-harvesting complexes of PSI (LHCI) and PSII (LHCII), and transfer of absorbed energy to photochemical reaction centers. LHCII plays an essential role in the regulation of photosynthesis, as well as in photoprotection [1-4]. The crystal structure of spinach (Spinacia oleracea) LHCII revealed that the basic structure and functional unit of the major antenna is a protein trimer with bound chlorophylls $a$ and $b$, carotenoides, and lipids [5]. The release of monomers from LHCII trimers was found

Address reprint requests to Professor Dr. Michael Przybylski, University of Konstanz, Laboratory of Analytical Chemistry and Biopolymer Structure Analysis, Department of Chemistry, Universitaetsstrasse 10, 78457 Konstanz, Germany. E-mail: michael.przybylski@uni-konstanz.de

* Present address: Department of Mathematics and Natural Sciences, University of Stavanger, 3046 Stavanger, Norway. during assembly [6] and disassembly of LHCII before its degradation upon excess light [7]. LHCII isolated from thylakoid membranes of Arabidopsis thaliana contains a mixture of 14 different light-harvesting chlorophyll $a / b$-binding proteins (Lhcb1-6 proteins and their isomers) with similar polypeptide sequences and similar structures and functions $[8,9]$. The Lhcb4-6 proteins and their isomers form a monomeric, minor LHCII antenna, whereas the Lhcb1-3 proteins and their isomers form the major LHCII antenna, composed of monomers and homo- or heterotrimers [1, 10-15]. The LHCII homotrimers are formed by Lhcb1, Lhcb2, but not by Lhcb3, although heterotrimers of different combinations of Lhcb1, Lhcb2, and Lhcb3 have been reported [10, 16]. Furthermore, it was found that heterotrimers containing Lhcb3 are not mobile, but remain tightly bound to PSII. The mobile part of LHCII is the basic unit of chloroplast state transitions-the shortterm tuning system in balancing the excitation energy between PSII and PSI. The minor LHC proteins are assigned to shuttle between PSI and PSII during state transitions, thereby acting as docking sites for the 
trimeric LHCII proteins [17, 18]. The supramolecular organization of LHCII, most likely arising from specific trimer-trimer contacts, has a significant effect on the pigment interactions in the complexes [19].

Gel electrophoretic methods such as Blue-native gel electrophoresis, isoelectric focusing (IEF), and sodium dodecylsulfate polyacrylamide gel electrophoresis (SDS-PAGE) for separation of LHCII complexes have been successfully applied in proteome analysis [20-25], but have shown insufficient resolution of LHC proteins with molecular masses between 22 and $25 \mathrm{kDa}$. For example, Lhcb1 and Lhcb2 protein isoforms, which are related to eight genes with similar pI and molecular masses [8], cannot be separated by SDS-PAGE. Electrospray-ionization (ESI) mass spectrometry combined with reversed-phase highperformance liquid chromatography (RP-HPLC) has been reported to be an effective tool to characterize PSI- and PSII-related membrane proteins and has been successfully used to separate and identify LHC protein isoforms [26-29]. Using quadrupole [29] and ion trap [28] mass analyzers, intact protein masses could be determined with standard deviations of 2-5 Da. These approaches, based on nonisotopically resolved related ion deconvolution using average masses from protein spectra, showed in some cases differences between measured and calculated masses higher than standard deviations [28], probably because of the complexity of mixtures of protein isoforms and the presence of post-translational modifications. The limitations of these approaches can be overcome by increased mass resolution and mass accuracy. Highly accurate mass determinations by Fourier transform ion cyclotron resonance mass spectrometry (FTICR-MS) allow direct and unambiguous identifications of protein mixtures, providing an efficient alternative to conventional protein separation and identification [30-32].

Under high-light conditions, oxygenic photosynthesis produces radicals and active oxygen species, such as superoxide, hydrogen peroxide, and hydroxyl radicals $[33,34]$. These reactive oxygen species are able to cause photo-oxidative damage of Photosystem II. Using protein carbonylation as a marker of oxidative damage in Arabidopsis [35], a drastic reduction of protein carbonyls after 20 days was observed, whereas carbonylation levels of Lhcb proteins were found to fluctuate, such that oxidation was mainly observed during the night. Mass spectrometric approaches based on accurate measurements of low-abundance oxidatively modified peptides, may significantly contribute to understanding the physiological functions of Lhcb proteins. In this study we have identified LHCII proteins and proteins associated with LHCII subcomplexes isolated from thylakoid membrane of $A$. thaliana leaves after light stress treatment, using high-resolution mass spectrometry for proteome analysis. LHCII subcomplexes were isolated by sucrose density gradient centrifugation and gel-filtration. RPHPLC and 2D gel electrophoresis (2-DE) separations were performed for proteome analysis using both ESI and matrix-assisted laser desorption/ionization (MALDI)-
FTICR-MS. The high-resolution mass spectrometric approaches enabled the identification of a series of LHCII proteins and their subcomplexes, and provided details of oxidative modifications. Several Lhcb1 and Lhcb2 proteins were identified to contain up to four protein isoforms that remained undetected by electrophoretic separation. These results provided crucial structure information of LHC protein isoforms during shortterm adaptation to light stress conditions, illustrating FTICR-MS as an efficient tool for identification of protein mixtures.

\section{Experimental}

\section{Plant Growth and Stress Conditions}

Arabidopsis thaliana L. ecotype Columbia were grown in a growth chamber on soil (Professional soil type $\mathrm{P}$, Gebr. Patzer $\mathrm{GmbH}$, Sinntal-Jossa, Germany) at $25^{\circ} \mathrm{C}$ at a photon flux density of $100 \mu \mathrm{mol}$ of photons $\mathrm{m}^{-2} \mathrm{~s}^{-1}$ at a light regime 8-h light/16-h dark. Light stress treatment was performed on mature leaves, detached from 4- to 5-week-old-plants, floated on water, and exposed to a light intensity of $2000 \mu \mathrm{mol}$ of photons $\mathrm{m}^{-2} \mathrm{~s}^{-1}$ for $3 \mathrm{~h}$ using a fluorescent light source (HQI-E bulb, 400 W/D; Osram, Regensburg-Burgweinting, Germany). The spectrum of the lamp covered a visible light region from 380 to $720 \mathrm{~nm}$. The temperature of the water was kept constant between 22 and $25^{\circ} \mathrm{C}$. Photon fluency rates were measured with a photometer (Skye, Techtum Laboratory $\mathrm{AB}$, Umeå, Sweden).

\section{Isolation of Monomeric and Trimeric LHCII Associated with PSII}

Thylakoid membrane preparations from light stressed 1-month-old $A$. thaliana leaves were performed according to Eshaghi and colleagues [36]. Thylakoid membranes were diluted to $0.5 \mathrm{mg} \mathrm{mL}{ }^{-1}$ chlorophyll in buffer containing $25 \mathrm{mM}$ MES (pH 6.0), $10 \mathrm{mM} \mathrm{NaCl}, 5$ $\mathrm{mM} \mathrm{MgCl}$, and $2 \mathrm{M}$ betaine supplemented with $20 \mathrm{mM}$ $n$-dodecyl- $\beta$-D-maltoside (DM) and disrupted by six strokes of a glass homogenizer for 10 to $20 \mathrm{~s}$. A $700 \mu \mathrm{L}$ aliquot of the homogenate was loaded on the top of a linear $0-8 \%(\mathrm{wt} / \mathrm{vol})$ sucrose density gradient in $2 \mathrm{M}$ betaine, $25 \mathrm{mM}$ MES ( $\mathrm{pH}$ 5.7), $10 \mathrm{mM} \mathrm{NaCl}, 5 \mathrm{mM}$ $\mathrm{CaCl}_{2}$, and $0.5 \%(\mathrm{wt} / \mathrm{vol}) \mathrm{DM}$ that was obtained by a freezing $\left(2 \mathrm{~h}\right.$ at $\left.-80^{\circ} \mathrm{C}\right)$ and thawing $\left(5-10 \mathrm{~min}\right.$ at $\left.25^{\circ} \mathrm{C}\right)$ cycle. After centrifugation at $27,000 \mathrm{rpm}$ for $12 \mathrm{~h}$ at $4{ }^{\circ} \mathrm{C}$ two main green bands were visible: the upper containing LHCII and the lower containing PSII-LHCII supercomplex [36]. The LHCII containing fractions were pooled, concentrated by size-exclusion centrifugation (vivaspin columns, exclusion size $10 \mathrm{kDa}$ ), and loaded on a gel-filtration column (Superdex 200, Amersham Biosciences, Uppsala, Sweden). The sample was eluted with the running buffer containing $100 \mathrm{mM} \mathrm{NaCl}, 50$ $\mathrm{mM}$ Tris $/ \mathrm{HCl}(\mathrm{pH} 8.0)$ and $0.05 \%$ (wt/vol) DM at the flow velocity of $0.3 \mathrm{~mL} \mathrm{~min}^{-1}$. The elution profile in 
terms of absorbance at $280 \mathrm{~nm}$ showed three major peaks, where peaks 1 and 2 contained LHCII (not shown). The fractions of peak 1 and peak 2 were pooled separately and concentrated by size-exclusion centrifugation as cited earlier. The protein concentration of each peak was determined according to Bradford [37]. Aliquots of $60 \mu \mathrm{g}$ protein were purified using the 2-D-Gel Clean-Up Kit (GE Healthcare, Munich, Germany) according to the manufacturer's protocol.

\section{Isolation of Trimeric LHCII Subcomplexes Associated with PSI Attributed to State Transition}

The thylakoids were prepared as described earlier. Thylakoid membranes were resuspended in water at a chlorophyll concentration of $0.8 \mathrm{mg} \mathrm{mL}^{-1}, 10 \%$ (wt/vol) $\mathrm{DM}$ was added to a final concentration of $0.9 \%$, and the mixture was incubated for $20 \mathrm{~min}$ on ice. The solubilized thylakoids were centrifuged at 20,000 $\mathrm{g}$ for $20 \mathrm{~min}$ and the clear supernatant (about $700 \mu \mathrm{L}$ ) was loaded on a $0-10 \%$ (wt/vol) linear sucrose density gradient containing $5 \mathrm{mM}$ Tricine $(\mathrm{pH} 8.0)$ and $0.05 \%(\mathrm{wt} / \mathrm{vol}) \mathrm{DM}$. The gradients were centrifuged at $170,000 \mathrm{~g}(32,000 \mathrm{rpm}$ in the SW40Ti rotor) for $17 \mathrm{~h}$ at $4{ }^{\circ} \mathrm{C}$. After centrifugation, the gradients showed two major green bands (the upper band contained the PSII complex and the lower band the PSI complex). The PSI-containing fractions were harvested, concentrated, and loaded on the Superdex 200 gelfiltration column. Separation was performed as described earlier. The elution profile monitored at $280 \mathrm{~nm}$ showed three major peaks, where only peak 2 contained LHCII. The peak 2 fractions were pooled, concentrated, and prepared for MS analysis according to the manual provided for the 2-D-Gel Clean-Up Kit.

\section{RP-HPLC Separation}

HPLC separations were carried out on a Bio-Rad 2700 HPLC system (Bio-Rad Laboratories, Hercules, CA, USA) equipped with a UV detector. Samples were loaded onto the column by an injection valve with a $200 \mu \mathrm{l}$ sample loop. Proteins were separated on a reversed-phase Vydac Protein C-4 column of $250 \times$ $4.6 \mathrm{~mm}$ inner diameter, packed with $5 \mu \mathrm{m}$ porous butyl silica (Vydac, Hesperia, CA, USA). All solutions were sonicated for $15 \mathrm{~min}$ in an ultrasonic bath (Transsonic 570, Elma, Germany) and degassed under vacuum. The column was preeqilibrated with $28 \%$ (vol:vol) acetonitrile solution containing $0.1 \%$ (vol: vol) trifluoroacetic acid (TFA) in Milli-Q water (Millipore, Bedford, MA, USA), and samples were eluted using a linear gradient from 28 to $64 \%$ (vol:vol) of acetonitrile in $0.1 \%$ (vol:vol) TFA in Milli-Q water in $45 \mathrm{~min}$. At the end of the run, the column was flushed for 10 min with $80 \%$ (vol:vol) acetonitrile in $0.1 \%$ (vol:vol) TFA.

\section{Electrophoretic Separation}

IEF was carried out with a Multiphor horizontal electrophoresis system (Amersham Biosciences) using $17 \mathrm{~cm}$ IPG strips ( $\mathrm{pH} 4-7$ linear), with the sample applied overnight using the in-gel rehydration method. Sample aliquots of $60 \mu \mathrm{g}$ were dissolved in rehydration solution containing 7 M urea, $2 \mathrm{M}$ thiourea, $4 \%$ (wt/vol) CHAPS, 0.3\% (wt/vol) dithiothreitol (DTT), 2\% (wt/vol) Servalyte 4-7, $40 \mathrm{mM}$ Tris-Base, and trace amounts of bromphenol blue. The rehydrated strip was run in the first dimension for about $30 \mathrm{kVh}$ at $20^{\circ} \mathrm{C}$. After the IEF step, the IPG strip was equilibrated for $40 \mathrm{~min}$ in $6 \mathrm{M}$ urea, $30 \%$ (wt/vol) glycerol, 2\% (wt/vol) SDS, $0.05 \mathrm{M}$ Tris- $\mathrm{HCl}$ ( $\mathrm{pH} 8.8), 1 \%$ (wt/vol) DTT, and a trace of bromphenol blue, then for $20 \mathrm{~min}$ in the same solution except that the DTT solution was replaced by $4.5 \%$ (wt/vol) iodoacetamide. The second-dimensional (2D) separations were carried out with a Protean II xi vertical electrophoresis system (Bio-Rad Laboratories) using 12\% SDS-PAGE self-made gels $(1.5 \times 200 \times 200 \mathrm{~mm})$. The strips were placed on the vertical gels; overlaid with $0.5 \%$ (wt/vol) agarose in SDS running buffer containing $25 \mathrm{mM}$ Tris- $\mathrm{HCl}, 192$ $\mathrm{mM}$ glycine, and $0.1 \%$ (wt/vol) SDS; and subjected to electrophoresis at $25 \mathrm{~mA} / \mathrm{gel}$ for $30 \mathrm{~min}$ and $40 \mathrm{~mA} /$ gel until the tracking dye reached the anodic end of the gel. Proteins were visualized by high-sensitivity colloidal Coomassie Blue and scanned using a Bio-Rad GS-710 calibrated imaging densitometer.

\section{Tryptic Digestion and Peptide Extraction}

RP-HPLC protein fractions were lyophilized, then 40 $\mu \mathrm{L}$ of a trypsin solution (12.5 $\mathrm{ng} \mu \mathrm{L}^{-1}$ trypsin in $50 \mathrm{mM}$ $\mathrm{NH}_{4} \mathrm{HCO}_{3}$ ) was added and incubated for $4 \mathrm{~h}$ at $37^{\circ} \mathrm{C}$. Gel spots were excised and destained using acetonitrile: $\mathrm{H}_{2} \mathrm{O}$ (wt/vol 3:2) and $50 \mathrm{mM}$ ammonium bicarbonate. The gel pieces were dehydrated with acetonitrile, dried in vacuo for $15 \mathrm{~min}$, and incubated for $45 \mathrm{~min}$ at $4{ }^{\circ} \mathrm{C}$ with $40 \mu \mathrm{L}$ of a trypsin solution (12.5 ng $\mu \mathrm{L}^{-1}$ trypsin in $50 \mathrm{mM} \mathrm{NH}_{4} \mathrm{HCO}_{3}$ ). After pulling off the reaction solution, $10 \mu \mathrm{L}$ of buffer without protease was added and the reaction continued for $20 \mathrm{~h}$ at $37^{\circ} \mathrm{C}$. The solution was then lyophilized and desalted using the C18ZipTip (Millipore).

\section{FTICR Mass Spectrometry and Database Search}

Mass spectrometric analysis was performed with a Bruker APEX II FTICR mass spectrometer (Bruker Daltonics, Billerica, MA, USA) equipped with an actively shielded 7 T superconducting magnet and a cylindrical infinity ICR analyzer cell. A Scout 100 MALDI source (Bruker Daltonik, Bremen, Germany) with pulsed in-source collision gas (argon) and in-source ion-accumulation capability was used for MALDI measurements [30]. A $20 \mathrm{mg} \mathrm{mL}^{-1}$ solution of 2,5-dihydroxybenzoic acid (DHB, Aldrich, Steinheim, Germany) in acetonitrile: $0.1 \%$ (vol:vol) TFA in Milli-Q (wt/vol, 2:1) was used as the matrix. A mixture of 
sample solution and matrix solution was prepared, applied on the AnchorChip 600 target, and allowed to dry at room temperature.

Nano-ESI-MS was performed using a self-modified APOLLO II source (Bruker Daltonik), with the needle tip placed a few millimeters offset in front of the entrance capillary. The voltage applied to the capillary entrance was set to 1000-1200 V. Mass spectra were deconvoluted using the DataAnalysis 3.2 software (Bruker Daltonik). Monoisotopic masses of singly charged ions were directly used for database search with MASCOT peptide mass fingerprinting as the search engine (http://www. matrixscience.com/search_form_select.html). The database used was NCBInr, a compilation of several databases including Swiss-Prot, PIR, PRF, PDB, and GenBank CDS translation. Search criteria for all experiments included preference in taxonomy for $A$. thaliana, one missed cleavage, oxidation of Met, His, and Trp as variable modifications, and a peptide search tolerance of $10 \mathrm{ppm}$. The fixed modification carbamidomethyl was set for 2-DE samples. Criteria for positive identification constituted three or more tryptic peptides, at least $30 \%$ sequence coverage, and a MOWSE score of $\geq 67$.

\section{Results}

\section{Identification of LHCII Intact Proteins and Their Oxidative Modifications}

LHC proteins associated with PSII were isolated from thylakoid membranes of $A$. thaliana leaves exposed at low-light conditions and after light stress treatment, separated by HPLC and analyzed by nano-ESI-FTICRMS. Mass spectrometric proteome analysis of intact proteins was combined with peptide mass fingerprinting of proteolytic peptide mixtures, by performing tryptic digestion in solution for each protein fraction. FTICR-MS enabled accurate mass determinations of intact light-harvesting proteins, but showed limitations attributed to the low ionization efficiencies of high molecular weight ions of hydrophobic proteins. The abundance of large-protein ions was found to be significantly suppressed in ESI-FTICR mass spectra relative to lipids, pigments, and small proteins. To reduce these suppression effects, HPLC protein fractions were lyophilized and redissolved in a mixture containing water, $60-75 \%$ acetonitrile, and $0.02-0.05 \%$ formic acid. Accurate mass determinations of Lhcb1-3 proteins were obtained by optimization of measurement conditions, including solvent composition and mass selective enhancement procedures for external ion accumulation in the $\mathrm{m} / \mathrm{z}$ range between 1000 and 1400 , similar to those described in Wong and Amster [38].

Figure 1a shows the HPLC separation of LHCII proteins after light stress treatment. The mass spectrum of the most abundant protein isoform related to Lhcb1.1-1.3 gene products is presented in Figure 1b. The protein spectrum in the $\mathrm{m} / \mathrm{z}$ range between 1000
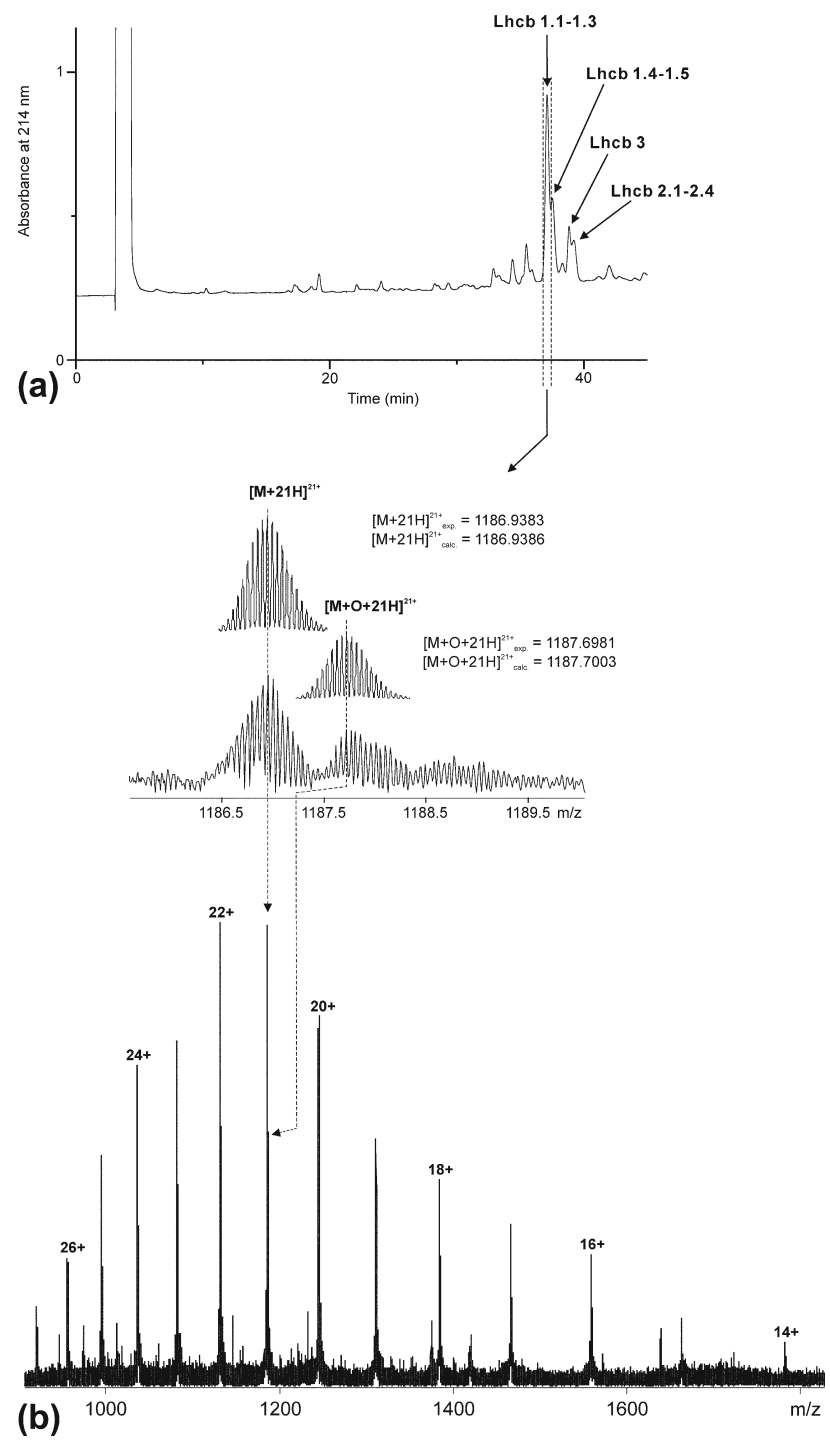

Figure 1. Light-harvesting proteins isolated from Arabidopsis thaliana thylakoid membranes, separated by RP-HPLC and identified by FTICR mass spectrometry. (a) Chromatographic separation of $200 \mu \mathrm{g}$ LHCII proteins after light stress treatment. (b) ESI-FTICR mass spectrum of intact Lhcb1.1-1.3 shows at least one protein modification by oxygen.

and 1400 showed no significant overlapping with other species and provided accurate mass determination. The series of peaks related to the $21+$ charged state of the intact protein showed the $[\mathrm{M}+21 \mathrm{H}]^{+}$ion as the most intensive signal, and an additional ion signal corresponding to the oxidized form $[\mathrm{M}+\mathrm{O}+21 \mathrm{H}]^{+}$. Additional modifications by oxidation, loss of water, and formation of sodium adducts cause isotopic distributions deviating from the calculated values. The protein sequence was additionally characterized by tryptic peptides and provided the identification of modifications by one, two, and three oxygen atoms and by $\mathrm{N}$-terminal acetylation. Using both mass spectrometric approaches, six major LHCII proteins were characterized. Because of the overlapping of several protein isoforms, the evaluation procedure based on accurate 
Table 1. Comparison of molecular masses of intact light-harvesting proteins of $A$. thaliana determined by ESI-FTICR-MS analysis after separation by HPLC with the protein masses expected from the DNA sequence

\begin{tabular}{|c|c|c|c|c|c|}
\hline $\begin{array}{l}\text { Protein } \\
\text { identified }^{a}\end{array}$ & $\begin{array}{l}\text { Measured } \\
\text { mass }^{\mathrm{b}}\end{array}$ & Molecular formula & $\begin{array}{l}\text { Calculated } \\
\text { mass }^{c}\end{array}$ & $\begin{array}{l}\text { Standard } \\
\text { deviation }\end{array}$ & Modifications \\
\hline \multirow[t]{2}{*}{ Lhcb1.1-1.3 } & $24,904.5729$ & $\mathrm{C}_{1139} \mathrm{H}_{1715} \mathrm{~N}_{293} \mathrm{O}_{327} \mathrm{~S}_{5}$ & $24,904.5585$ & 0.0699 & Acetyl (N-term.) \\
\hline & $24,920.5736$ & $\mathrm{C}_{1139} \mathrm{H}_{1715} \mathrm{~N}_{293} \mathrm{O}_{328} \mathrm{~S}_{5}$ & $24,920.5535$ & 0.0829 & Acetyl (N-term.); $+[\mathrm{O}]$ \\
\hline \multirow[t]{2}{*}{ Lhcb1.4 } & $24,775.4570$ & $\mathrm{C}_{1133} \mathrm{H}_{1700} \mathrm{~N}_{290} \mathrm{O}_{327} \mathrm{~S}_{5}$ & $24,775.4319$ & 0.2306 & Acetyl (N-term.) \\
\hline & $24,791.3117$ & $\mathrm{C}_{1133} \mathrm{H}_{1700} \mathrm{~N}_{290} \mathrm{O}_{328} \mathrm{~S}_{5}$ & $24,791.4269$ & 0.2661 & Acetyl (N-term.); $+[\mathrm{O}]$ \\
\hline \multirow[t]{2}{*}{ Lhcb1.5 } & $24,915.5950$ & $\mathrm{C}_{1141} \mathrm{H}_{1716} \mathrm{~N}_{293} \mathrm{O}_{327} \mathrm{~S}_{5}$ & $24,915.5633$ & 0.1669 & Acetyl (N-term.) \\
\hline & $24,931.5797$ & $\mathrm{C}_{1141} \mathrm{H}_{1716} \mathrm{~N}_{293} \mathrm{O}_{328} \mathrm{~S}_{5}$ & $24,931.5582$ & 0.2164 & Acetyl (N-term.); +[O] \\
\hline \multirow[t]{2}{*}{ Lhcb2.1-2.3 } & $24,943.5150$ & $\mathrm{C}_{1148} \mathrm{H}_{1716} \mathrm{~N}_{288} \mathrm{O}_{327} \mathrm{~S}_{5}$ & $24,943.5512$ & 0.1122 & Acetyl (N-term.) \\
\hline & $24,959.5791$ & $\mathrm{C}_{1148} \mathrm{H}_{1716} \mathrm{~N}_{288} \mathrm{O}_{328} \mathrm{~S}_{5}$ & $24,959.5461$ & 0.2470 & Acetyl (N-term.); +[O] \\
\hline \multirow[t]{2}{*}{ Lhcb2.4 } & $24,931.5609$ & $\mathrm{C}_{1146} \mathrm{H}_{1712} \mathrm{~N}_{288} \mathrm{O}_{326} \mathrm{~S}_{6}$ & $24,931.4966$ & 0.1056 & Acetyl (N-term.) \\
\hline & $24,947.4670$ & $\mathrm{C}_{1146} \mathrm{H}_{1712} \mathrm{~N}_{288} \mathrm{O}_{327} \mathrm{~S}_{6}$ & $24,947.4915$ & 0.2495 & Acetyl (N-term.); +[O] \\
\hline \multirow[t]{2}{*}{ Lhcb3 } & $24,280.3134$ & $\mathrm{C}_{1123} \mathrm{H}_{1675} \mathrm{~N}_{281} \mathrm{O}_{313} \mathrm{~S}_{5}$ & $24,280.2800$ & 0.1001 & \\
\hline & $24,296.2524$ & $\mathrm{C}_{1123} \mathrm{H}_{1675} \mathrm{~N}_{281} \mathrm{O}_{314} \mathrm{~S}_{5}$ & $24,296.2749$ & 0.1349 & $+[\mathrm{O}]$ \\
\hline
\end{tabular}

aprotein identifications were performed by peptide mass fingerprinting of in-solution digested protein fractions. Additional assignments for protein isoforms with high sequence similarity were made by using the mass of an intact protein.

${ }^{b}$ Measured mass of an uncharged protein based on the most intensive isotopic peaks from the five most intensive charge states.

${ }^{c}$ Calculated mass of the most intensive isotopic peak of an uncharged assigned gene product.

mass determination using most intensive isotopic peaks was used. By this procedure, the mass determination accuracy for protein isoforms was determined to be $<10$ ppm. The masses of the most abundant isotopic peaks of the identified LHCII protein isoforms are presented in Table 1.

Based on the intact protein measurements, no significant quantitative changes in the LHCII compositions were found after light stress treatment, compared with the low-light conditions. Only a small decrease of the amounts of Lhcb1.4, Lhcb2.1-2.3, and Lhcb2.4 isoforms was observed under high-light conditions. Thus, it was not possible to quantify the changes in oxidative modifications arising from the light stress treatment.

\section{Separation and Identification of Proteins in LHCII Subcomplexes under Light-Stress Conditions}

Three LHCII subcomplexes were isolated by sucrose density gradient centrifugation and gel-filtration using a strategy similar to that described in Eshaghi et al. [36] and van Roon et al. [39]. Proteins of each LHCII subcomplex were separated by 2-DE, visualized with Coomassie staining, and analyzed by MALDI-FTICR-
MS. Monoisotopic masses of singly charged ions from the MALDI-MS data were applied to database search using MASCOT peptide fingerprinting. Figure 2 shows the separation of proteins from LHCII subcomplexes 1 (Figure 2a) and 2 (Figure 2b) associated with PSII, and of LHCII subcomplex 3 (Figure 2c) associated with PSI attributed to state transition.

The 2-DE at the pI region of 4.0 to 5.5 revealed the presence of 14 protein spots with molecular masses between 20 and $35 \mathrm{kDa}$ for the LHCII subcomplex 1, including five isoforms of Lhcb1 (Lhcb1.1-1.5); four isoforms of Lhcb2 (Lhcb2.1-2.4); one isoform each of Lhcb3, Lhcb5, and Lhcb6; and two different fibrillins (Figure 2a). The LHCII subcomplex 2 contained five isoforms of Lhcb1 (Lhcb1.1-1.5), four isoforms of Lhcb2 (Lhcb2.1-2.4), and Lhcb3. The minor antenna proteins Lhcb4, Lhcb5, Lhcb6, and fibrillins were not detected in this subcomplex (Figure 2b). The LHCII subcomplex 3 consisted of five isoforms of Lhcb1 (Lhcb1.1-1.5) and four isoforms of Lhcb2 (Lhcb2.1-2.4) in addition to two fibrillins (Figure 2c).

MALDI-FTICR-MS was optimized in sensitivity in the mass range 1000 to $4000 \mathrm{Da}$ using a strategy described in Mize and Amster [40]. In-source accumu-

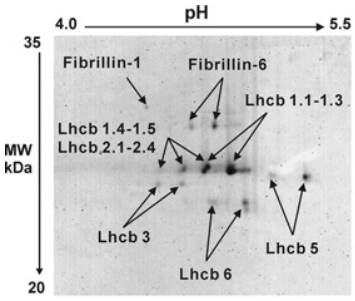

(a)

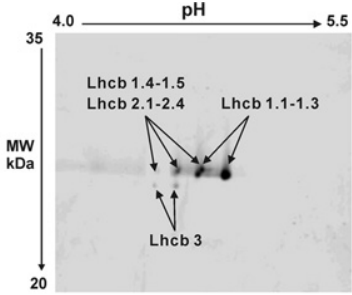

(b)

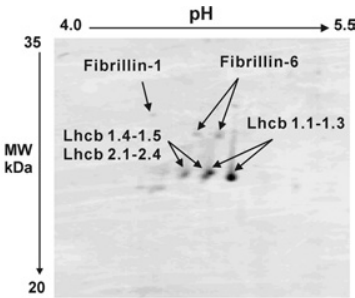

(c)

Figure 2. Light-harvesting complexes isolated from Arabidopsis thaliana thylakoid membranes and separated by 2D gel electrophoresis. Protein spots were excised from the gel, digested with trypsin as described in the experimental section. (a) LHCII subcomplex 1. (b) LHCII subcomplex 2. (c) LHCII subcomplex 3 associated with PSI attributed to state transitions. 
lation of ions was used for up to 30 laser shots under collisional cooling of ions, by averaging mass spectra of up to 128 scans. Homogeneous co-crystallization of the matrix/sample mixture on the AnchorChip target was found suitable to reduce space charge effects, causing small frequency shifts and differences in ion abundances from scan to scan. Using a standard peptide mixture (five measurements) the maximum mass error after optimization was $<5$ ppm and the standard deviation $<2.5$ ppm (32 scans, accumulation of 20 laser shots). A mass tolerance of 10 ppm was used for the database search. For all identified proteins a sequence coverage of $>30 \%$ and a MOWSE score $>67$ were obtained.

\section{Identification of Lhcb1 and Lhcb2 Protein Isoforms by FTICR-MS}

Several protein mixtures consisting of Lhcb1 and Lhcb2 isoforms were identified from unresolved gel spots (see Figure 2). Seven different light-harvesting proteins were identified in 11 gel spots and up to four proteins related to different genes were identified in a single gel spot. As an example, Figure 3 shows the MALDI-FTICR mass spectrum and identification of proteins encoded by $l h c b 1$ and $l h c b 2$ genes in the gel spot at pI 4.7; MW 25 $\mathrm{kDa}$. The mass spectra provided peptide measurements within a wide dynamic range $(\sim 1000)$ and identifications for four Lhcb1 and Lhcb2 proteins by the database

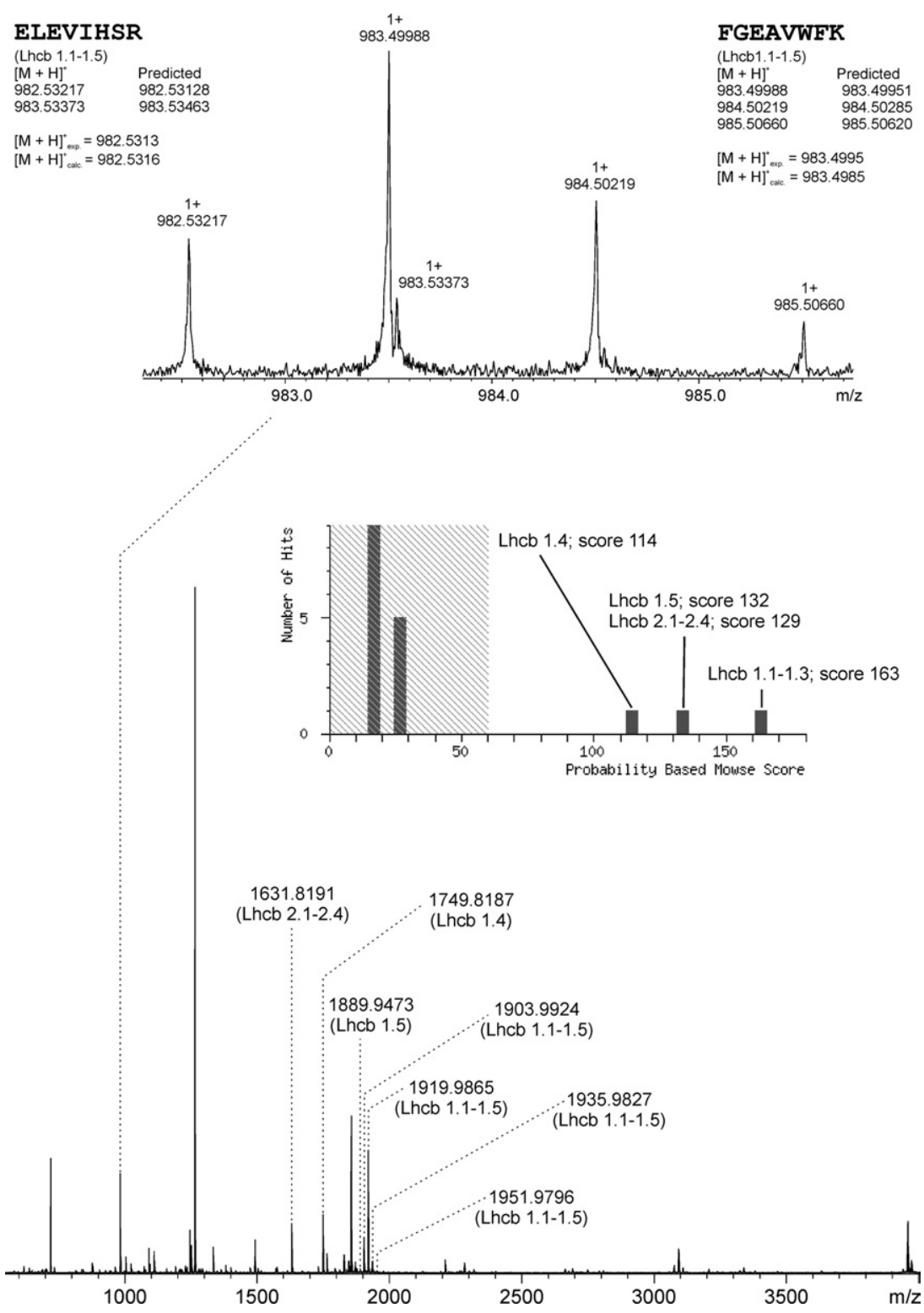

Figure 3. Identification of a mixture of Lhcb1.1-1.3, Lhcb1.4, Lhcb1.5, and Lhcb2.1-2.4 protein isoforms in protein spot at pI 4.7; MW $25 \mathrm{kDa}$ (Figure 2a) in LHCII subcomplex 1 isolated from Arabidopsis thaliana thylakoid membranes. The mass peaks containing specific sequences and modifications illustrated in Figures 4 and 5 are underlined. 
Table 2. Peptide identifications of Lhcb1 and Lhcb2 protein isoforms in trimeric subunits of LHCII isolated from A. thaliana thylakoid membranes ${ }^{a}$

\begin{tabular}{|c|c|c|c|c|}
\hline Protein & $m / z_{\text {exp. }}(\mathrm{Da})$ & $m / z_{\text {calc. }}(\mathrm{Da})$ & $\Delta m(\mathrm{ppm})$ & Sequence \\
\hline Lhcb1.4 & 1877.9260 & 1877.9140 & 6.4 & KASKPTGPSGSPWYGSDR \\
\hline Lhcb1.5 & 1889.9473 & 1889.9504 & 1.6 & TVAKPKGPSGSPWYGSDR \\
\hline Lhcb1.4 & 1749.8187 & 1749.8183 & 0.2 & ASKPTGPSGSPWYGSDR \\
\hline Lhcb1.1-1.5 & 1976.9919 & 1976.9751 & 4.8 & ASKPTGPSGSPWYGSDRVK \\
\hline Lhcb1.1-1.3; 1.5 & 1265.5533 & 1265.5545 & 0.9 & GPSGSPWYGSDR \\
\hline Lhcb1.1-1.3; 1.5 & 1492.7218 & 1492.7179 & 2.6 & GPSGSPWYGSDRVK \\
\hline Lhcb1.4; 1.5 & 3967.7927 & 3967.7972 & 1.2 & YLGPFSGEPPSYLTGEFPGDYGWDTAGLSADPETFAR \\
\hline Lhcb1.4; 1.5 & 3983.7873 & 3983.7921 & 1.3 & YLGPFSGEPPSYLTGEFPGDYGWDTAGLSADPETFAR + $1[0]$ \\
\hline Lhcb1.1-1.3 & 3957.8019 & 3957.7765 & 6.4 & YLGPFSGESPSYLTGEFPGDYGWDTAGLSADPETFAR \\
\hline Lhcb1.1-1.3 & 3973.7920 & 3973.7714 & 5.2 & YLGPFSGESPSYLTGEFPGDYGWDTAGLSADPETFAR + 1[0] \\
\hline Lhcb1.1-1.5; 2.1-2.4 & 1252.6770 & 1252.6756 & 1.2 & NRELEVIHSR \\
\hline Lhcb1.1-1.5; 2.1-2.4 & 982.5313 & 982.5316 & 0.3 & ELEVIHSR \\
\hline Lhcb1.1-1.5 & 1903.9924 & 1903.9920 & 0.3 & WAMLGALGCVFPELLAR \\
\hline Lhcb1.1-1.5 & 1919.9865 & 1919.9870 & 0.3 & WAMLGALGCVFPELLAR $+1[0]$ \\
\hline Lhcb1.1-1.5 & 1935.9827 & 1935.9820 & 0.4 & WAMLGALGCVFPELLAR $+2[0]$ \\
\hline Lhcb1.1-1.5 & 1951.9796 & 1951.9770 & 1.3 & WAMLGALGCVFPELLAR + 3[0] \\
\hline Lhcb1.1-1.5; 2.1-2.4 & 983.4995 & 983.4985 & 1.0 & FGEAVWFK \\
\hline Lhcb1.4; 1.5 & 3631.7764 & 3631.7689 & 2.1 & VAGDGPLGEAEDLLYPGGSFDPLGLATDPEAFAELK \\
\hline Lhcb1.1-1.5 & 3092.5235 & 3092.5224 & 0.3 & GPLENLADHLADPVNNNAWAFATNFVPGK \\
\hline Lhcb1.1-1.5 & 3108.5206 & 3108.5173 & 1.1 & GPLENLADHLADPVNNNAWAFATNFVPGK $+1[0]$ \\
\hline Lhcb2.1-2.4 & 1960.0131 & 1960.0287 & 7.9 & TVKSTPOSIWYGPDRPK \\
\hline Lhcb2.1-2.4 & 1631.8191 & 1631.8176 & 0.9 & STPQSIWYGPDRPK \\
\hline Lhcb2.1-2.4 & 1893.9710 & 1893.9601 & 5.8 & WAMLGALGCTFPEILSR \\
\hline Lhcb2.1-2.4 & 1909.9670 & 1909.9551 & 6.2 & WAMLGALGCTFPEILSR + 1[O] \\
\hline Lhcb2.1-2.4 & 2292.1828 & 2292.1879 & 2.6 & WAMLGALGCTFPEILSRNGVK \\
\hline Lhcb2.1-2.4 & 2308.2008 & 2308.1829 & 7.8 & WAMLGALGCTFPEILSRNGVK $+1[0]$ \\
\hline Lhcb2.1-2.4 & 1381.7343 & 1381.7263 & 5.8 & NGVKFGEAVWFK \\
\hline Lhcb2.1-2.4 & 2321.1927 & 2321.1933 & 0.3 & NGPLAMFSMFGFFVQAIVTGK \\
\hline Lhcb2.1-2.4 & 1994.0183 & 1994.0278 & 4.8 & LAMFSMFGFFVQAIVTGK \\
\hline
\end{tabular}

asee Figure 2a, gel spot at pl 4.7; MW $25 \mathrm{kDa}$.

search with accuracies of 29 matching peptide masses $<8 \mathrm{ppm}$. Because of the sequence homology of the identified proteins, three ions at $\mathrm{m} / \mathrm{z} 982.5313, \mathrm{~m} / \mathrm{z}$ 983.4995, and $\mathrm{m} / \mathrm{z} 1252.6770$ matched all identified proteins. At the mass resolution used $(\sim 100,000)$ two adjacent peaks with a mass difference of $34 \mathrm{ppm}$ could be separated. One isotope peak $(\mathrm{m} / \mathrm{z}$ 983.4999) was assigned as the first monoisotopic peak of the sequence ELEVIHSR, whereas the second at $\mathrm{m} / \mathrm{z} 983.5337$ was assigned as the second isotopic peak of the sequence FGEAVWFK. Tryptic fragments of proteins identified in the Lhcb1/Lhcb2 gel spot at $25 \mathrm{kDa}$, pI 4.7, are summarized in Table 2. Four protein isoforms were differentiated by specific peptides, with a standard deviation of $2.6 \mathrm{ppm}$ of the mass determination error. Proteins Lhcb2.1-2.4 and proteins Lhcb1.1-1.3 are represented by the same tryptic peptides and therefore their isoforms could not be differentiated. Accurate mass determinations of specific peptides with significant differences in relative abundances at $\mathrm{m} / \mathrm{z} 1749.8187$ (8.6\%), $m / z 1889.9473(0.7 \%)$, and $m / z 1631.8176(7.3 \%)$ provided the identification of the isoforms Lhcb1.4, Lhcb1.5, and Lhcb2.1-2.4, respectively (Figure 4). No differences in the relative abundance of these proteins were detected in LHCII subcomplexes 1 and 2. In contrast, the LHCII subcomplex 3 showed a decreased level of Lhcb1.4 and Lhcb2 isoforms in comparison to Lhcb1.1-1.3.

Modifications by oxidation were found in all identified LHCII proteins. Figure 5 shows the partial sequence ${ }^{70}$ WAMLGALGCVFPELLAR ${ }^{86}$ of the Lhcb1.1-1.3 isoform that contained oxidative modifications most likely at Trp and/or Met residues (see Figure 3 and Table 2). The ions at $\mathrm{m} / \mathrm{z}$ 1903.9924, 1919.9865, 1935.9827, and $\mathrm{m} / \mathrm{z}$ 1951.9796 represent the unmodified peptide (70-86) of Lhcb1.1-1.3, and the peptide with modifications by one, two, and three oxygen atoms, respectively. The accurate peptide masses and the mass differences between the modified peptides were in good agreement with the calculated masses of the corresponding proteolytic peptides.

\section{Discussion}

In this study we report three LHCII subcomplexes from light stress-treated leaves of $A$. thaliana that differ in their protein composition. Subcomplex 1 consists of three major antenna proteins Lhcb1, Lhcb2, Lhcb3, and their isomers, two minor antenna proteins, Lhcb5 and Lhcb6, and two different fibrillins. Subcomplex 2 contains the major antenna proteins Lhcb1, Lhcb2, Lhcb3, and their isomers, but is lacking the minor antenna proteins and fibrillins. 


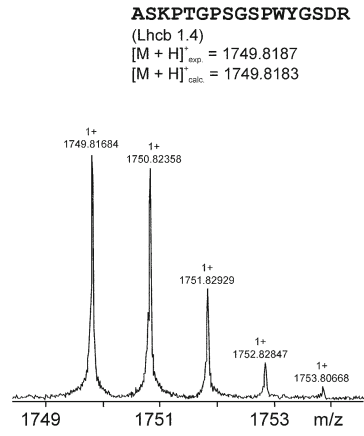

(a)

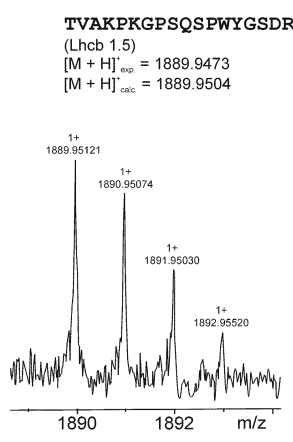

(b)

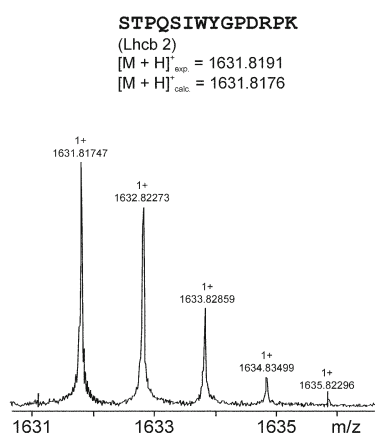

(c)

Figure 4. Experimental and calculated masses of peptide sequences of Lhcb1.4 (a), Lhcb1.5 (b), and Lhcb2 (c) protein isoforms identified by MALDI-FTICR-MS in protein spot at pI 4.7; MW $25 \mathrm{kDa}$ (see Figure 3).

The presence of Lhcb3 in both subcomplexes suggests that both are tightly bound to PSII. Subcomplex 3 was found to consist of Lhcb1 and Lhcb2 and their isomers, and is associated with PSI.

From the results of the present study, we conclude that in the present subcomplex preparations the LHCII subcomplex 1 represents the monomeric antenna, subcomplex 2 the trimeric antenna thightly bound to PSII, and subcomplex 3 a mobile trimeric LHCII that migrates from PSII to PSI attributed to state transition (see Figure 6). The subcomplex compositions identified by mass spectrometry agree with compositions in previous reports derived from gel chromatographic and electrophoretic separations $[6,7,10-13]$. State transition in higher plants is a short-term adaptation to changes in light intensity [18, 41]. Interestingly, the amounts of Lhcb1.4-1.5 and Lhcb2.1-2.4 proteins relative to Lhcb1.1-1.3 proteins changed significantly in the mobile LHCII trimer associated with PSI, compared to the tightly bound LHCII monomers and trimers, as shown

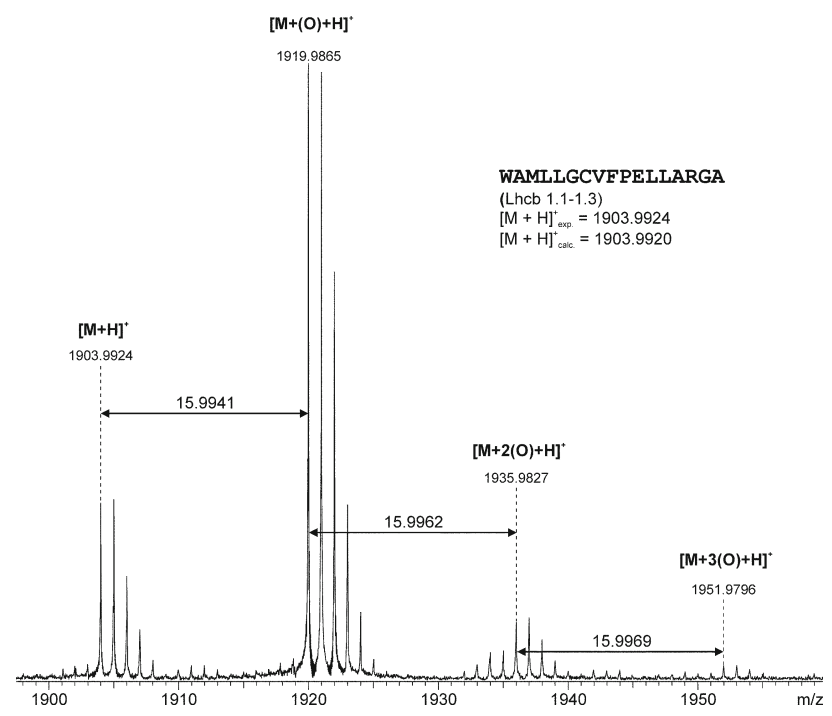

Figure 5. Identification of protein modification by one, two, and three oxygen atoms by MALDI-FTICR-MS in protein spot at pI 4.7; MW 25 kDa (see Figure 3). in Figure 2. This can be explained either by reorganization of subcomplexes or by higher mobilities of trimers composed of Lhcb1.1-1.3.

Both the tightly bound LHCII subcomplex 1 and the mobile subcomplex 3 contain fibrillins, although their amounts were much lower in the mobile complex, whereas no fibrillin was detected in subcomplex 2. Two different fibrillins were identified: Fibrillin-1 (At4g0420) was present in gel spot at $\mathrm{pI} 4.4$; MW $28 \mathrm{kDa}$, whereas Fibrillin-6 (At3g23400) was identified in the gel spots at pI 4.6; MW $27 \mathrm{kDa}$ and at pI 4.7; MW $27 \mathrm{kDa}$. Fibrillins are plastid-lipid associated proteins (PAPs) that belong to the plastoglobulin family and are induced by oxidative stress [42, 43]. Fibrillins contain a conserved lipocalin signature involved in transport of small hydrophobic molecules (e.g., porphyrins) [24]. Thus, the presence of fibrillins in LHCII subcomplexes might be connected to the release and transfer of pigments from photodamaged Lhcb proteins.

It is known that under light stress conditions chlorophylls bound to LHC proteins can form triplet states upon light absorption that in turn can react with molecular oxygen and form singlet oxygen [44, 45]. Different reactive oxygen species can also be generated dur-

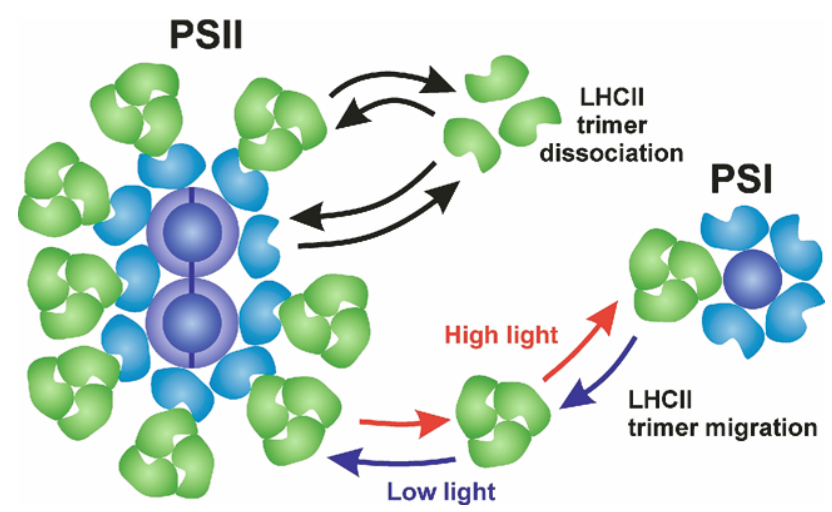

Figure 6. Structure model of the redistribution of LHCII subcomplexes in PSII and PSI after state transition. Dark blue, core complex; light blue, monomeric antenna pool; green, trimeric antenna pool. 
ing water splitting in the oxygen-evolving complex associated with PSII. We found that the fragment of the first $\alpha$-helical part of Lhcb1.1-1.3 (70-86) was modified by one, two, and three oxygen atoms, respectively, most likely by oxidation at Met-72 and Trp-70. Oxidation of Met may be an indicator of a change in protein conformation [46, 47]. The adjacent residue Arg-69 has been reported to be involved in chlorophyll binding in Lhcb1 by neutralization of Asp und Glu residues through ionic interaction [48]. Thus, oxidation of Trp-70 [49] might influence protein-chlorophyll binding and lead to conformational changes in the organization of LHCII. In addition to the fragment (70-86), several other sites in the LHCII proteins were found modified by oxidation, of which the oxidation of Met residues may have occurred in-gel, in agreement with the identification of the nonoxidized form in Lhcb proteins. Other oxidative modifications might be related to processes such as photoprotection, photodamage, or repair of damaged proteins and will be the subject of further investigations.

\section{Conclusion}

In conclusion, proteome analysis of light-harvesting subcomplexes revealed a variety of Lhcb isoforms playing an important role in the organization, reorganization, and mobility of these subcomplexes. The identification of several proteins modified by oxidation may be related to protective or other physiological functions. Because of its high resolution and mass accuracy, FTICR-MS provided direct and unambiguous identifications of proteins and protein mixtures, thus presenting an efficient approach for high-performance proteomics. We have compared and combined in this study the utility of accurate intact protein mass determinations and peptide mass fingerprinting for the characterization of LCHII isoforms, and modifications by oxidation and N-terminal acetylation were identified. Using 2-DE combined with MALDI-FTICR-MS peptide mass fingerprinting, we identified high- and low-abundance proteins in different LHC subcomplexes. MALDIFTICR-MS, optimized in sensitivity and dynamic range, was found to be an effective tool for the analysis of low-abundance proteins and provided the identification of proteins in complex biological mixtures such as in tryptic digests from unseparated gel spots. Although Lhcb1.1-1.5 and Lhcb2.1-2.4 protein isoforms with similar pI (4.5-5.0) and MW (25 kDa) could not be separated, identifications of different isoforms were obtained based on accurate mass measurements of tryptic peptides. For the Lhcb1.1-1.5 and Lhcb2.1-2.4 protein isoforms, identifications were even obtained from unseparated gel spots, illustrating the efficiency of FTICR-MS to the analysis of protein mixtures.

\section{Acknowledgments}

This work has been supported in part by the Deutsche Forschungsgemeinschaft (Biopolymer-MS and Proteomics, 175/6-1 to M.P.; and
AD92/1-3 to I.A.). The authors thank Reinhold Weber for expert assistance with high-resolution mass spectrometry procedures and Roswitha Miller-Sulger for biochemical preparations.

\section{References}

1. Standfuss, J.; Terwisscha van Scheltinga, A. C.; Lamborghini, M. Kuhlbrandt, W. Mechanisms of Photoprotection and Nonphotochemical Quenching in Pea Light-Harvesting Complex at $2.5 \AA$ Resolution. EMBO J. 2005, 24, 919-928.

2. Horton, P.; Ruban, A. Molecular Design of the Photosystem II LightHarvesting Antenna: Photosynthesis and Photoprotection. J. Exp. Bot. 2005, 56, 365-373.

3. Minagawa, J.; Takahashi, Y. Structure, Function and Assembly of Photosystem II and Its Light-Harvesting Proteins. Photosynth. Res. 2004, 82, 241-263.

4. van Grondelle, R.; Novoderezhkin, V. I. Energy Transfer in Photosynthesis: Experimental Insights and Quantitative Models. Phys. Chem. Chem. Phys. 2006, 8, 793-807.

5. Liu, Z.; Yan, H.; Wang, K.; Kuang, T.; Zhang, J.; Gui, L.; An, X.; Chang, W. Crystal Structure of Spinach Major Light-Harvesting Complex at $2.72 \AA$ A Resolution. Nature 2004, 428, 287-292.

6. Dreyfuss, B. W.; Thornber, J. P. Assembly of the Light-Harvesting Complexes (LHCs) of Photosystem II (Monomeric LHC IIb Complexes Are Intermediates in the Formation of Oligomeric LHC IIb Complexes). Plant Physiol. 1994, 106, 829-839.

7. Garab, G.; Cseh, Z.; Kovács, L.; Rajagopal, S.; Várkonyi, Z.; Wentworth M.; Mustardy, L.; Der, A.; Ruban, A. V.; Papp, E.; Holzenburg, A.; Horton, P. Light-Induced Trimer to Monomer Transition in the Main Light-Harvesting Antenna Complex of Plants: Thermo-optic Mechanism. Biochemistry 2002, 41, 15121-15129.

8. Jansson, S. A Guide to the Lhc Genes and Their Relatives in Arabidopsis/IT>. Trends Plant Sci. 1999, 4, 236-240.

9. Caffarri, S.; Croce, R.; Cattivelli, L.; Bassi, R. A Look within LHCII: Differential Analysis of the Lhcb1-3 Complexes Building the Major Trimeric Antenna Complex of Higher-Plant Photosynthesis. Biochemistry 2004, 43, 9467-9476.

10. Standfuss, J.; Kuhlbrandt, W. The Three Isoforms of the Light-Harvesting Complex II: Spectroscopic Features, Trimer Formation, and Functional Roles. J. Biol. Chem. 2004, 279, 36884-36891.

11. Danielsson, R.; Suorsa, M.; Paakkarinen, V.; Albertsson, P. A.; Styring, S.; Aro, E. M.; Mamedov, F. Dimeric and Monomeric Organization of Photosystem II. Distribution of Five Distinct Complexes in the Different Domains of the Thylakoid Membrane. J. Biol. Chem. 2006, 281, 1424114249.

12. Kirchhoff, H.; Tremmel, I.; Haase, W.; Kubitscheck, U. Supramolecular Photosystem II Organization in Grana Thylakoid Membranes: Evidence for a Structured Arrangement. Biochemistry 2004, 43, 9204-9213.

13. Linnanto, J.; Martiskainen, J.; Lehtovuori, V.; Ihalainen, J.; Kananavicius, R.; Barbato, R.; Korppi-Tommola, J. Excitation Energy Transfer in the LHC-II Trimer: A Model Based on the New 2.72 A Structure. Photosynth. Res. 2006, 87, 267-279.

14. Hobe, S.; Forster, R.; Klingler, J.; Paulsen, H. N. Proximal Sequence Motif in Light-Harvesting Chlorophyll a/b-Binding Protein Is Essential for the Trimerization of Light-Harvesting Chlorophyll $a / b$ Complex. Biochemistry 1995, 34, 10224-10228.

15. Kuttkat, A.; Hartmann, A.; Hobe, S.; Paulsen, H. The C-Terminal Domain of Light-Harvesting Chlorophyll-a/b-Binding Protein Is Involved in the Stabilisation of Trimeric Light-Harvesting Complex. Eur. J Biochem. 1996, 242, 288-292.

16. Jackowski, G.; Kacprzak, K.; Jansson, S. Identification of Lhcb1/Lhcb2/ Lhcb3 Heterotrimers of the Main Light-Harvesting Chlorophyll a/bProtein Complex of Photosystem II (LHC II). Biochim. Biophys. Acta 2001, 1504, 340-345.

17. Takahashi, H.; Iwai, M.; Takahashi, Y.; Minagawa, J. Identification of the Mobile Light-Harvesting Complex II Polypeptides for State Transitions in Chlamydomonas reinhardtii. Proc. Natl. Acad. Sci. U.S.A. 2006 103, 477-482.

18. Jensen, P. E.; Bassi, R.; Boekema, E. J.; Dekker, J. P.; Jansson, S.; Leister, D.; Robinson, C.; Scheller, H. V. Structure, Function and Regulation of Plant Photosystem I. Biochim. Biophys. Acta 2007, 1767, 335-352.

19. Lambrev, P. H.; Várkonyi, Z.; Krumova, S.; Kovács, L.; Miloslavina, Y. Holzwarth, A. R.; Garab, G. Importance of Trimer-Trimer Interactions for the Native State of the Plant Light-Harvesting Complex II. Biochim. Biophys. Acta 2007, 1767, 847-853.

20. Aro, E. M.; Suorsa, M.; Rokka, A.; Allahverdiyeva, Y.; Paakkarinen, V.; Saleem, A.; Battchikova, N.; Rintamaki, E. Dynamics of Photosystem II: A Proteomic Approach to Thylakoid Protein Complexes. J. Exp. Bot. 2005, 56, 347-356.

21. Heinemeyer, J.; Eubel, H.; Wehmhoner, D.; Jansch, L.; Braun, H. P. Proteomic Approach to Characterize the Supramolecular Organization of Photosystems in Higher Plants. Phytochemistry 2004, 65, 1683-1692.

22. Suorsa, M.; Regel, R. E.; Paakkarinen, V.; Battchikova, N.; Herrmann, R. G.; Aro, E. M. Protein Assembly of Photosystem II and Accumulation of Subcomplexes in the Absence of Low Molecular Mass Subunits PsbL and PsbJ. Eur. J. Biochem. 2004, 271, 96-107. 
23. Friso, G.; Giacomelli, L.; Ytterberg, A. J.; Peltier, J. B.; Rudella, A.; Sun, Q.; Wijk, K. J. In-Depth Analysis of the Thylakoid Membrane Proteome of Arabidopsis thaliana Chloroplasts: New Proteins, New Functions, and a Plastid Proteome Database. Plant Cell 2004, 16, 478-499.

24. Ytterberg, A. J.; Peltier, J. B.; van Wijk, K. J. Protein Profiling of Plastoglobules in Chloroplasts and Chromoplasts. A Surprising Site for Differential Accumulation of Metabolic Enzymes. Plant Physiol. 2006, 140, 984-997.

25. Hippler, M.; Klein, J.; Fink, A.; Allinger, T.; Hoerth, P. Towards Functional Proteomics of Membrane Protein Complexes: Analysis of Thylakoid Membranes from Chlamydomonas reinhardtii. Plant J. 2001, 28, 595-606.

26. Timperio, A. M.; Huber, C. G.; Zolla, L. Separation and Identification of the Light Harvesting Proteins Contained in Grana and Stroma Thylakoid Membrane Fractions. J. Chromatogr. A. 2004, 1040, 73-81.

27. Timperio, A. M.; Zolla, L. Investigation of the Lateral Light-Induced Migration of Photosystem II Light-Harvesting Proteins by Nano-High Performance Liquid Chromatography Electrospray Ionization Mass Spectrometry. J. Biol. Chem. 2005, 280, 28858-28866.

28. Zolla, L.; Timperio, A. M.; Walcher, W.; Huber, C. G. Proteomics of Light-Harvesting Proteins in Different Plant Species. Analysis and Comparison by Liquid Chromatography-Electrospray Ionization Mass Spectrometry Photosystem II. Plant Physiol. 2003, 131, 198-214.

29. Gomez, S. M.; Nishio, J. N.; Faull, K. F.; Whitelegge, J. P. The Chloroplast Grana Proteome Defined by Intact Mass Measurements from Liquid Chromatography Mass Spectrometry. Mol. Cell. Proteomics 2002, 1, 46-59.

30. Baykut, G.; Jertz, R.; Witt, M. Matrix-Assisted Laser Desorption/ Ionization Fourier Transform Ion Cyclotron Resonance Mass Spectrometry with Pulsed In-Source Collision Gas and In-Source Ion Accumulation. Rapid Commun. Mass Spectrom. 2000, 14, 1238-1247.

31. Witt, M. Fuchser, J. Baykut, G. Fourier Transform Ion Cyclotron Resonance Mass Spectrometry with NanoLC/Microelectrospray Ionization and Matrix-Assisted Laser Desorption/Ionization: Analytical Performance in Peptide Mass Fingerprint Analysis. J. Am. Soc. Mass Spectrom. 2003, 14, 553-561.

32. Conrads, T. P.; Anderson, G. A.; Veenstra, T. D.; Pasa-Tolic, L.; Smith, R. D. Utility of Accurate Mass Tags for Proteome-wide Protein Identification. Anal. Chem. 2000, 72, 3349-3354.

33. Nield, J.; Redding, K.; Hippler, M. Remodeling of Light-Harvesting Protein Complexes in Chlamydomonas in Response to Environmental Changes. Eukaryot. Cell 2004, 3, 1370-1380.

34. Asada, K. Production and Scavenging of Reactive Oxygen Species in Chloroplasts and Their Functions. Plant Physiol. 2006, 141, 391-396.

35. Johansson, E.; Olsson, O.; Nystrom, T. Progression and Specificity of Protein Oxidation in the Life Cycle of Arabidopsis thaliana. J. Biol. Chem. 2004, 279, 22204-22208.
36. Eshaghi, S.; Andersson, B.; Barber, J. Isolation of a Highly Active PSII-LHCII Supercomplex from Thylakoid Membranes by a Direct Method. FEBS Lett. 1999, 446, 23-26.

37. Bradford, M. M. A Rapid and Sensitive Method for the Quantitation of Microgram Quantities of Protein Utilizing the Principle of Protein-Dye Binding. Anal. Biochem. 1976, 72, 248-254.

38. Wong, R. L.; Amster, I. J. Combining Low and High Mass Ion Accumulation for Enhancing Shotgun Proteome Analysis by Accurate Mass Measurement. J. Am. Soc. Mass Spectrom. 2006, 17, 205-212.

39. van Roon, H.; van Breemen, J. F.; de Weerd, F. L.; Dekker, J. P. Boekema, E. J. Solubilization of Green Plant Thylakoid Membranes with $n$-Dodecyl-Alpha, D-Maltoside. Implications for the Structural Organization of the Photosystem II, Photosystem I, ATP Synthase and Cytochrome b6f Complexes. Photosynth. Res. 2000, 64, 155-166.

40. Mize, T. H.; Amster, I. J. Broad-Band Ion Accumulation with an Internal Source MALDI-FTICR-MS. Anal. Chem. 2000, 72, 5886-5891.

41. Kouril, R.; Zygadlo, A.; Arteni, A. A.; de Wit, C. D.; Dekker, J. P.; Jensen, P. E.; Scheller, H. V.; Boekema, E. J. Structural Characterization of a Complex of Photosystem I and Light-Harvesting Complex II of Arabidopsis thaliana. Biochemistry 2005, 44, 10935-10940.

42. Brehelin, C.; Kessler, F.; van Wijk, K. J. Plastoglobules: Versatile Lipoprotein Particles in Plastids. Trends Plant Sci. 2007, 12, 260-226.

43. Langenkamper, G.; Manac'h, N.; Broin, M.; Cuine, S.; Becuwe, N. Kuntz, M.; Rey, P. Accumulation of Plastid Lipid-Associated Proteins (Fibrillin/CDSP34) upon Oxidative Stress, Ageing and Biotic Stress in Solanaceae and in Response to Drought in Other Species. J. Exp. Bot. 2001, 52, 1545-1554.

44. Anderson, J. M.; Chow, W. S. Structural and Functional Dynamics of Plant Photosystem II. Philos. Trans. R. Soc. Lond. B Biol. Sci. 2002, 357, 1421-1430; discussion, 1469-1470.

45. Vogt, W. Oxidation of Methionyl Residues in Proteins: Tools, Targets, and Reversal. Free Radic. Biol. Med. 1995, 18, 93-105.

46. Chao, C. C.; Ma, Y. S.; Stadtman, E. R. Modification of Protein Surface Hydrophobicity and Methionine Oxidation by Oxidative Systems. Proc. Natl. Acad. Sci. U.S.A. 1997, 94, 2969-2974.

47. Dalle-Donne, I.; Rossi, R.; Giustarini, D.; Gagliano, N.; Di Simplicio, P.; Colombo, R.; Milzani, A. Methionine Oxidation as a Major Cause of the Functional Impairment of Oxidized Actin. Free Radic. Biol. Med. 2002, 32 927-937.

48. Kuhlbrandt, W.; Wang, D. N.; Fujiyoshi, Y. Atomic Model of Plant Light-Harvesting Complex by Electron Crystallography. Nature 1994, $367,614-621$.

49. Kunz, L.; Zeidler, U.; Haegele, K.; Przybylski, M.; Stark, G. Photodynamic and Radiolytic Inactivation of Ion Channels Formed by Gramicidin A: Oxidation and Fragmentation. Biochemistry 1995, 34, 1189511903. 